\title{
Prevalence of celiac disease in multiple sclerosis
}

\author{
Luis Rodrigo ${ }^{1 *}$, Carlos Hernández-Lahoz², Dolores Fuentes', Noemí Alvarez ${ }^{1}$, Antonio López-Vázquez³ \\ Segundo González ${ }^{3}$
}

\begin{abstract}
Background: Celiac disease (CD) is a common systemic disease related to a permanent intolerance to gluten and is often associated with different autoimmune and neurological diseases. Its mean prevalence in the general population is $1-2 \%$ worldwide. Our aim was to study the prevalence of celiac disease in a prospective series of Multiple Sclerosis (MS) patients and their first-degree relatives.

Methods: We analyzed the prevalence of serological, histological and genetic CD markers in a series of 72 MS patients and in their 126 first-degree relatives, compared to 123 healthy controls.

Results: Tissue IgA-anti-transglutaminase-2 antibodies were positive in 7 MS patients (10\%), compared to 3 healthy controls (2.4\%) $(p<0.05)$. OR: 5.33 (CI-95\%: 1.074-26.425). No differences were found in HLA-DQ2 markers between MS patients (29\%) and controls (26\%) (NS).

We detected mild or moderate villous atrophy (Marsh III type) in duodenal biopsies, in 8 MS patients (11.1\%). We also found a high proportion of CD among first-degree relatives: 23/126 (32\%). Several associated diseases were detected, mainly dermatitis 41 (57\%) and iron deficiency anemia in 28 (39\%) MS patients. We also found in them, an increased frequency of circulating auto-antibodies such as anti-TPO in 19 (26\%), ANA in 11 (15\%) and AMA in 2 (3\%).

Conclusions: We have found an increased prevalence of CD in 8 of the 72 MS patients (11.1\%) and also in their first-degree relatives (23/126 [32\%]). Therefore, increased efforts aimed at the early detection and dietary treatment of $C D$, among antibody-positive MS patients, are advisable.
\end{abstract}

\section{Background}

Multiple Sclerosis (MS) is a chronic disease of unknown etiology, characterized by the presence of disseminated demyelinating lesions in the central nervous system (CNS), and associated with autoimmunity. Activated, potentially autoimmune, $T$ cells cross the blood-brain barrier and produce inflammatory plaques and axonal loss in the brain, spinal cord or optic nerves. The end result is the accumulation of gliosis and demyelination and areas in the CNS. MS affects about $1 \%$ of the population worldwide.

Mainly occurs in young people, more often women. The Relapsing-Remitting form of Multiple Sclerosis (RRMS) makes up $80 \%$ of the total number of MS cases and is characterized by intermittent episodes of relapses and prolonged remissions. Clinically, patients display

\footnotetext{
* Correspondence: Irodrigosaez@gmail.com

'Gastroenterology, Hospital Universitario Central de Asturias (HUCA), C/

Celestino Villamil, s. n’., 33006. Oviedo, Spain

Full list of author information is available at the end of the article
}

episodes of acute neurological dysfunction, followed by recovery and a symptom-free interval until the next outbreak. These recurrent events eventually lead to more permanent neurological disabilities. Using an experimental model of autoimmune encephalitis as a starting point, immunomodulatory and immunosuppressive therapies have then proved effective in preventing relapses in MS patients, especially when performed early in the course of the disease [1-7].

Celiac disease (CD) is a systemic autoimmune disorder characterized by permanent intolerance to gluten in genetically predisposed individuals. The genetic basis for gluten intolerance is located in the region of chromosome 6 coding for HLA class-II [8-11]. Some patients with RRMS show high levels of anti-tissue transglutaminase-2 (TGt-2) antibodies, which is an important serological marker in the diagnosis of the disease [12]. Based on this observation and on the possible association of MS with other autoimmune processes, we have applied a
C Biomed Central

() 2011 Rodrigo et al; licensee BioMed Central Ltd. This is an Open Access article distributed under the terms of the Creative Commons Attribution License (http://creativecommons.org/licenses/by/2.0), which permits unrestricted use, distribution, and reproduction in any medium, provided the original work is properly cited. 
specific protocol for the systematic assessment of $C D$ in a population of RRMS patients.

\section{Methods \\ Patients}

We conducted a prospective observational study of a consecutive series of 80 patients suffering from wellestablished and clinically definite MS. They were previously diagnosed with RRMS and checked up at an outpatient clinic for demyelinating disorders within the Department of Neurology at the Central University Hospital of Asturias (HUCA). This is an urban tertiary hospital located in Northern Spain, serving an area with a population of 250,000. Patients were enrolled during a one-year period (January-December 2006). Of the initial 80 RRMS a total of 72, were included in this study (the other 8 didn't complete the study protocol).

MS patients with primary or secondary progressive forms of the disease (PP or SP) were not included in the study, because most of these patients were very physically disabled, in wheelchairs, and it would have been very inconvenient for them to attend the necessary check-ups.

We also included in this study a total of 126 firstdegree relatives of the 72 RRMS patients. We compared the findings with a control group of 123 marrow blood donors of the same area.

The study was approved by the Research and Ethics Committee of the HUCA, following the principles included in the modified Declaration of Helsinki.

All RRMS patients in this series were diagnosed on the basis of medical history, neurological examination and paraclinical positive tests, including Magnetic Resonance Imaging (MRI), Cerebrospinal fluid (CSF) and Visually Evoked Potentials (VEP), according to the 2005 McDonald criteria [13]. All cases also met the spatial and temporal dissemination criteria.

\section{Clinical parameters}

At the time of inclusion in the study, RRMS patients underwent a brain and spine MRI with intravenous injection of $0.2 \mathrm{ml} / \mathrm{kg}$ body weight of gadolinium (Magnevist $^{\mathrm{TM}} 0.5 \mathrm{mmol} / \mathrm{ml}$ ) as a contrast agent to assess uptake. All subjects were studied and monitored by the same neurologist (CHL), who followed-up with them at least twice a year.

With regard to treatment, 48 of the patients were receiving immunomodulatory therapy. Four patients received interferon beta-1a ( $30 \mathrm{mcg}$. IM/weekly), while 39 received interferon beta-1a (22-44 mcg. SQ/3 days per week) or interferon beta-1b (250 mcg SQ, on alternate days). Five other patients received glatiramer acetate $(20 \mathrm{mg}$ $\mathrm{SQ} /$ daily). No patient received immunosuppressive therapy. The remaining 24 patients were not treated.
During relapses, subjects received hospital inpatient treatment with methylprednisolone (500-1000 mg IV/ day for 3-5 days), except those with sensitive outbreaks, who were treated as outpatients with oral prednisone at a dose of $1 \mathrm{mg} / \mathrm{kg} /$ day for 1 week. In both cases, the initial steroid therapy was progressively decreased throughout the treatment period.

\section{MS studies}

MS disease activity was assessed by measuring the annual relapse rates and the EDSS (Expanded Disability Status Scale). The initial relapse rate value was calculated as the average over the two years preceding the admission into the study. EDSS was assessed at the time of admission into the study, leaving at least one month between the most recent episode and the EDSS assessment. The data on the number of relapses, their annual rate, and EDSS were updated at every follow-up visit, during the study period.

\section{CD studies}

All patients were invited to participate on a voluntary basis in a screening test for $\mathrm{CD}$ associated with the diagnosed RRMS. In 90\% of the cases, the patients accepted. These subjects were referred to an outpatient Gastroenterology clinic specialized in the study of small intestine diseases, which was located in the same hospital. All consenting patients were evaluated by the same gastroenterologist (LR). Every patient underwent a series of analytical assessments, which included serological and CD genetic markers, together with an upper GI endoscopy with multiple duodenal biopsies.

\section{Laboratory tests}

Complete blood count tests including erythrosedimentation rates (ESRs) were performed with an automated hematology analyzer (Cell-DYN 3500 model R Abbott); complete coagulation panels were also carried out with a coagulation analyzer (ACL 3000, Menarini). Normal hemoglobin values were in the $12-14 \mathrm{~g} / \mathrm{dl}$ range, and cases of iron deficiency anemia were defined according to WHO criteria, i.e. $\mathrm{Hb}<$ $13 \mathrm{~g} / \mathrm{dl}$ in males and $<12 \mathrm{~g} / \mathrm{dl}$. in females. Normal white blood cell count was set between $4-10 \times 10^{3} / \mathrm{L}$ and normal platelet count was between $130-400 \times 10^{3} / \mathrm{L}$.

Comprehensive analytical biochemistry tests were performed on the blood samples, including analyses of the following parameters: iron metabolism, including serum iron levels; transferrin saturation index (TSI) and serum ferritin. Normal values of serum iro were in the $60-140 \mathrm{mcg} / \mathrm{ml}$ range, whereas the normal ferritin values were $13-150 \mathrm{ng} / \mathrm{ml}$. The TSI was considered normal, for values ranging between $25-45 \%$.

We also assessed liver function tests (LFTs) including alkaline phosphatase (AP), aspartate aminotransferase 
(AST), alanine aminotransferase (ALT), gamma glutamyl transpeptidase (GGT) and bilirubin. The following measurements were also performed: total serum calcium, folate and vitamin B-12, serum creatinine, total cholesterol (with normal values in the $150-240 \mathrm{mg} / \mathrm{dl}$ range), high density lipoprotein (HDL), low density lipoprotein (LDL) and triglycerides, urea, glucose, total protein, albumin and acute phase reactants such as CRP (C-reactive protein). The normal values of AST and ALT were between 1-31 U/L. Immunoglobulins (IgG, IgA, IgM) were quantified by nephelometry, and circulating levels of the thyroid stimulating hormone (TSH, normal range: 0.25-5.0 mU/L) and the thyroid hormones (T3 and T4) were also evaluated. Finally, an urinalysis with microscopic examination of sediment was performed. Serum levels of triglycerides and total cholesterol were determined using enzymatic tests and values were expressed in $\mathrm{mg} / \mathrm{dl}$. All analytical studies were completed on a Hitachi Modular automated analyzer SXA-PPBD (Roche) using enzymatic or kinetic methods.

\section{Serological markers of autoimmunity}

The presence of anti-nuclear antibodies (ANA) and antithyroid antibodies like anti-peroxidase (anti-TPO) were measured routinely, and in those cases with altered LFTs, the anti-mitochondrial antibody (AMA) was also assessed. The determination of ANAs, anti-TPO and AMAs, was conducted by indirect immunofluorescence assay on the Hep-20-10 cell line (Euro-Immun, Lübeck, Germany).

\section{Serological markers of celiac disease}

Tissue transglutaminase 2 (TGt-2) anti-IgA antibody count was the only marker used; quantification was achieved with the aid of a commercially available ELISA kit (Phadia Diagnostics, Upsala, Sweden). We reported as positive those tests with values $>2 \mathrm{U} / \mathrm{ml}$, a threshold considered to allow for a greater sensitivity in the general population [14], and which we had already confirmed in a previous comparative study of several commercial kits [15]

\section{Genetic markers}

To assess genetic susceptibility to CD two HLA-class II markers, the HLA-DQ2 (DQA1*0501 and DQB1*0201) and the HLA-DQ8 (DQA1*0301 and DQB1*0302) were assayed, by using a Polymerase Chain Reaction (PCR) with a commercially available kit (Protrans ${ }^{\circledR}$ HLA Celiac Disease Domino System, Protrans, Ketsch, Germany).

\section{Duodenal biopsy studies}

Upon approval of informed consent procedures by the Hospital Ethics Committee, an upper endoscopy with multiple duodenal biopsies (at least 4) was performed in all the patients included in the study. Samples were routinely stained with Hematoxylin-Eosin (HE) and with immunohistochemical CD3-specific stains, which are routinely used to verify the presence of intraepithelial lymphocytes (IEL). IELs were in turn quantified per 100 epithelial cells.

Duodenal biopsies were studied by two expert pathologists and classified into the following types: Stage 0: Histologically normal duodenum; Stage 1: Increased IEL infiltration with a total count $>25 \%$ of epithelial cells; Stage 2: hyperplasia of crypts and/or diffuse chronic inflammatory infiltrate at the level of the lamina propria; Stage 3: villous atrophy, subdivided into three categories: a) mild, b) moderate, and c) severe, according to the anatomo-pathological classification for $\mathrm{CD}$ screening previously described by Marsh in 1992 [16] and later modified by Oberhuber et al. [17] in 1999.

We used neither gastric biopsies nor breath testing to routinely determine the presence of Helicobacter pylori infection in our patients.

\section{Statistics}

Descriptive statistics were used on continuous parameters: calculation of means, standard deviations, and observed ranges. For qualitative variables, percentages were used in the analyses. We determined the Chi-square test for these categorical variables between non-paired groups, using the exact Fisher's test when necessary. The odds ratio and CI-95\% were also calculated. A p value < 0.05 , was considered as statistically significant.

\section{Results}

Of the 72 RRMS patients included into the study, 60 (83\%) were women, giving a female-male ratio of $5 / 1$. Their ages ranged from 24 to 58 years old. The mean duration of RRMS, the clinical activity, the number of patients receiving any type of immunomodulatory treatment, and the percentage of fertility are shown in Table 1.

The clinical indicators of MS and the results of several paraclinical tests are presented, including the presence of oligoclonal bands (OCB) in the CSF, visual evoked potentials (VEP) and somato-sensorial evoked potentials

Table 1 Clinical and demographic characteristics of RRMS patients $(\mathbf{n}=\mathbf{7 2})$

\begin{tabular}{lc}
\hline Females, $\mathrm{n}(\%)$ & $60(83)$ \\
\hline Age in years, mean \pm SD (range) & $43 \pm 10(24-61)$ \\
\hline RRMS duration in years, mean \pm SD (range) & $11 \pm 6(1-30)$ \\
\hline Annual relapse rate, mean \pm SD (range) & $1.1 \pm 0.4(0-2)$ \\
\hline EDSS*, mean \pm SD, [median], (range) & $1.7 \pm 1.1,[2],(0-5)$ \\
Immunomodulatory therapy, n(\%) & $44(67)$ \\
\hline They have had children, $\mathrm{n}(\%)$ & $32(53)$ \\
\hline
\end{tabular}

EDSS $^{*}=$ Expanded Disability Status Scale; SD = Standard Deviation. 
(SSEP), together with MRI findings at the time of inclusion in the study (Table 2).

Overall, the mean values for the different blood chemistry analyses performed on the population were normal, showing a significant dispersion and wide ranges.

The frequency of serological and genetic markers was compared to the findings in the control group. Histological duodenal biopsies were made in only RRMS patients and their results are shown in Table 3.

Age, sex, anti-TGt2 values, genetic and histologic characteristics of the eight cases diagnosed with simultaneous celiac disease, which showed slight increased serologic levels of TGt-2 and diverse degrees of villous atrophy are shown in Table 4.

The clinical characteristics of the eight patients with associated celiac disease refered to the presence of gastrointestinal symptoms were found in 6 (75\%); weight loss was observed in 1 patient (12.5\%). The main neurologic form was myelitis in $4(50 \%)$ and mixed in 4 $(50 \%)$. No ataxia forms of presentation were found. Three patients $(37.5 \%)$ were treated with interferonbeta. We found 2 patients (25\%) with ANAs (+). All these data are shown in Table 5.

All the celiac patients were put on a gluten free diet and all of them improved considerably both with respect to the gastrointestinal and to the neurological symptomatology in the follow-up period.

The only differential parameter between celiacs and non-celiacs was the age of onset of MS, that was younger $(35 \pm 7$ y.o $)$ in the former and older $(44 \pm 10 \mathrm{y}$. o) in the latter $(\mathrm{p}<0.05)$

We have observed a high frequency of several different related diseases. Dermatitis: 41 (57\%); Iron deficiency anemia: 28 (39\%); Liver function test alterations: 18 (25\%); Recurrent urinary tract infections: $12(17 \%)$; Osteoporosis: 4 (6\%) (Figure 1).

It is significant that a high prevalence of $C D$ was found in first-degree relatives of RRMS patients, with 23 (32\%) positive cases among the 126 studied individuals.

We also discovered a variety of associated circulating auto-antibodies in these patients, the most frequently

Table 2 Paraclinical tests of RRMS patients $(n=72)$

\begin{tabular}{|c|c|}
\hline CSF oligoclonal bands' ${ }^{1}$, n (\%) & $51(71)$ \\
\hline Altered VEPs ${ }^{2}+, \mathrm{n}(\%)$ & $46(64)$ \\
\hline Altered SEPs ${ }^{3}+, \mathrm{n}(\%)$ & $52(72.2)$ \\
\hline T1-weighted gadolinium-enhanced MRI ${ }^{4}$ lesions, $\mathrm{n}^{5}$ (\%) & $25(35)$ \\
\hline T2-MRI supratentorial lesions, mean \pm SD (range) & $4.6 \pm 6.5(6-30)$ \\
\hline T2-MRI infratentorial lesions, mean \pm SD (range) & $1.6 \pm 2.5(0-10)$ \\
\hline T2-MRI medullar lesions, mean \pm SD (range) & $1.2 \pm 1.4(0-6)$ \\
\hline
\end{tabular}

Table 3 CD markers in RRMS and controls

\begin{tabular}{lccc}
\hline $\mathrm{CD}^{1}$ markers & $\begin{array}{c}\text { RRMS } \\
(\mathbf{n}=\mathbf{7 2})\end{array}$ & $\begin{array}{c}\text { Controls } \\
(\mathbf{n}=\mathbf{1 2 3})\end{array}$ & $\mathbf{p}$ \\
\hline Increased anti-tTG2 & \\
\hline HLA-DQ2 ${ }^{3}(+), \mathrm{n}[\%]$ & $7(10)$ & $3(2.4)$ & $<0.05$ \\
\hline $\mathrm{HLA}-\mathrm{DQ8}(+), \mathrm{n}(\%)$ & $21(29)$ & $32(26)$ & $\mathrm{NS}^{5}$ \\
\hline Villous atrophy (Marsh 3), n (\%) & $8(11)$ & $17(14)$ & $\mathrm{NS}$ \\
\hline${ }^{1} \mathrm{CD}=$ Celiac Disease; ${ }^{2}$ Anti-tTG2 $=$ Anti-tissue transglutaminase-2 antibodies \\
${ }^{3} \mathrm{HLA}=$ Human leukocyte antigens; ${ }^{4} \mathrm{ND}=$ Not done; ${ }^{5} \mathrm{NS}=$ Not significant.
\end{tabular}

observed being anti-thyroid peroxidase (anti-TPO) antibodies, followed by anti-nuclear antibodies (ANA), and, less frequently, anti-mitochondrial antibodies (AMA). Their percentages are shown in Figure 2.

\section{Discussion}

Our data support the hypothesis that there is an increased prevalence of serological markers of $C D$ in RRMS patients (OR: 5.33; CI-95\%: 1.07-26.45). Nevertheless, we have not found any differences between the DQ2 and DQ8 genetic markers in the patient and control groups. We also discovered several duodenal lesions in 21 (29\%) of RRMS patients and mild villous atrophy in $8(11.1 \%)$ of them.

All of these findings, together with the high prevalence of CD in first-degree relatives (32\%) support a frequent association with gluten intolerance in RRMS patients.

These results have been observed by other authors from Israel [18]. The specific role of serological gluten markers in the pathogenesis of multiple sclerosis remains uncertain. MS is a disorder presumed to be of autoimmune nature, characterized by a chronic demyelinating inflammatory process which affects the CNS, but its etiology remains unknown. MS affects mainly Caucasians, predominantly women, and in most cases, appears in young adulthood. Genetic, immunopathologic and environmental factors

Table 4 Demographic, serological, genetic and histological characteristics of the eight patients exhibiting an associated CD

\begin{tabular}{ccccccc}
\hline Patients & Sex & Age & $\begin{array}{c}\text { Anti-tTG2 (U/ } \\
\text { ml) }\end{array}$ & $\begin{array}{c}\text { HLA- } \\
\text { DQ2 }\end{array}$ & $\begin{array}{c}\text { HLA- } \\
\text { DQ8 }\end{array}$ & Marsh $^{2}$ \\
\hline 1 & $F^{1}$ & 33 & 187 & $(+)$ & $N^{3}$ & $3 b$ \\
\hline 2 & $F$ & 28 & 10.3 & $(+)$ & $N D$ & $3 a$ \\
\hline 3 & $F$ & 25 & 8.5 & $(+)$ & $N D$ & $3 a$ \\
\hline 4 & $F$ & 27 & 4.2 & $(+)$ & $N D$ & $3 a$ \\
\hline 5 & $F$ & 26 & 9.4 & $(+)$ & $N D$ & $3 b$ \\
\hline 6 & $F$ & 29 & 2.8 & $(+)$ & $N D$ & $3 a$ \\
\hline 7 & $F$ & 23 & 3.6 & $(-)$ & $(+)$ & $3 a$ \\
\hline 8 & $F$ & 34 & 1.8 & $(+)$ & $N D$ & $3 a$ \\
\hline
\end{tabular}

${ }^{1} \mathrm{~F}=$ Female; ${ }^{2}$ Marsh = Histological classification of duodenal biopsies for $\mathrm{CD}$; ${ }^{3} \mathrm{ND}=$ Not done. 
Table 5 Clinical characteristics of the eight patients with associated CD

\begin{tabular}{llccccc}
\hline Patients & Gastrointestinal symptoms & Weight loss & Neurological syndrome & IFN-beta treatment & Dermatitis & ANA (+) \\
\hline 1 & Diarrhea & Yes & Myelitis & No & Yes & No \\
\hline 3 & None & No & Myelitis & No & No & Yes \\
\hline 4 & GERD+Constipation & No & Optical neuritis & No & No & No \\
\hline 5 & Constipation & No & Encephalitis & No & Yes & No \\
\hline 6 & GERD & No & Myelitis & Yes & No \\
\hline 7 & GERD+Constipation & No & Encephalitis & No & No & No \\
\hline 8 & None & No & Optical neuritis & Yes & Yes & No \\
\hline
\end{tabular}

GERD = Gastroesophageal Reflux Disease; ANA = Antinuclear antibody test.

contribute to its complex multifactorial pathogenesis, via mechanisms that remain poorly understood [19].

Celiac disease (CD) is a systemic autoimmune disorder with a well-defined etiology. It appears as a consequence of permanent gluten intolerance, which occurs in genetically predisposed individuals. CD affects primarily, but not exclusively, the small intestine. It can start at any age, both during childhood or adolescence, and it often appears in adulthood [9]. Currently known genetic markers are excellent predictors of propensity for $\mathrm{CD}$, but a small proportion of patients $(5-10 \%)$ are negative for both DQ2 and DQ8, suggesting that other genetic markers of $\mathrm{CD}$ are yet to be described. Good candidates are additional HLA class I markers, including MICA and MICB, as well as other non-HLA markers detected by GWAS studies [20].

Until about two decades ago, CD was considered a rare disease, but it has now been shown to be very common, with a fairly uniform worldwide distribution and an average prevalence of $1-2 \%$, although this disease is often underestimated, and therefore under-diagnosed [21].

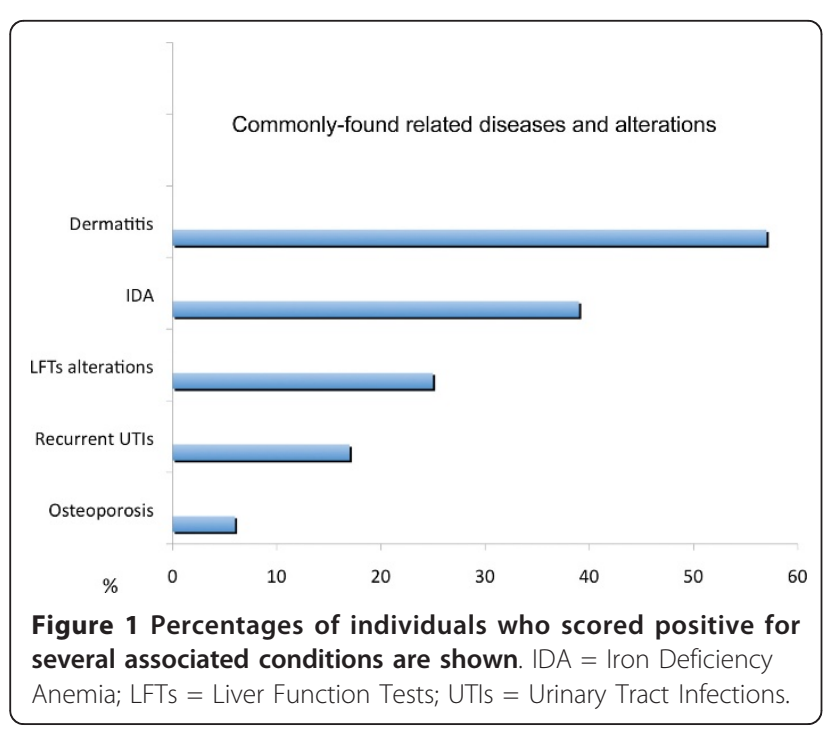

Individuals predisposed to $\mathrm{CD}$ exhibit inappropriate immune response to peptides derived from wheat, barley, and rye prolamins. Although several immunogenic epitopes are present in gluten, the most powerful is a peptide of 33 amino acids (residues 57-89), contained in the $\alpha$-gliadin fraction of gluten. This peptide is rich in proline and glutamine residues and appears to be primarily responsible for gluten toxicity, making it the main pathogenic factor linked to the occurrence of the disease [22].

During the pathogenesis leading to the appearance of lesions in the small intestine, gliadin peptides cross the intestinal epithelium and, at the level of the submucosa, they are modified by tissue transglutaminase (TGt2). Deamidated peptides are presented to dendritic cells with HLA-DQ2/DQ8 receptors, which in turn present them to CD4+ cells which, when activated, release cytokines such as Interferon-gamma (IFN- $\gamma$ ) and other mediators of inflammation. The characteristic histological changes, inflammation and/or villous atrophy, occur in the small intestinal mucosa $[23,24]$.

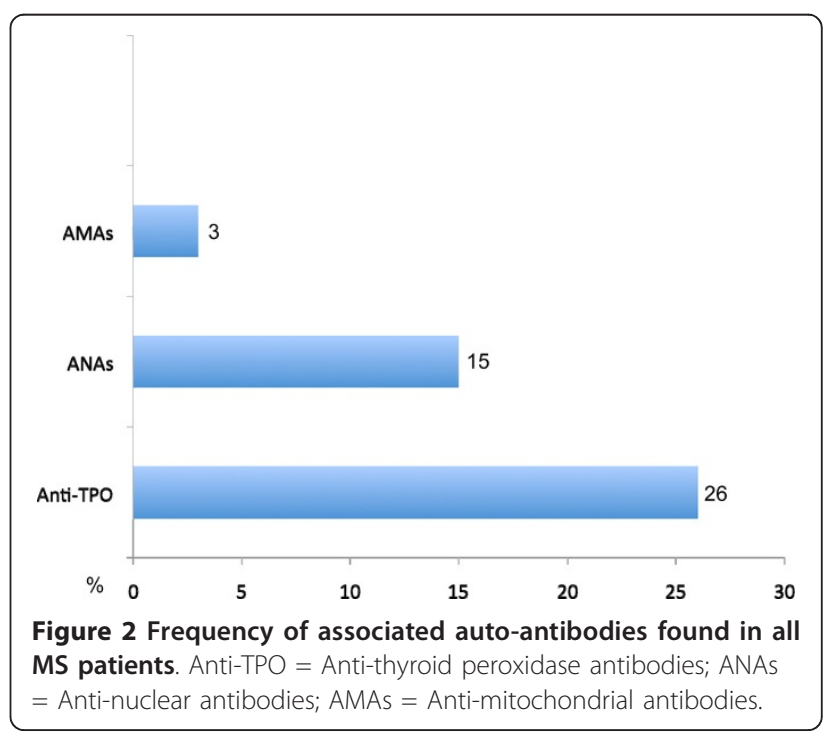


In 1997, Dieterich et al. reported that tissue transglutaminase type 2 (TGt-2) is the self-antigen that reacts against endomysium antibodies (EMA). This must to be evaluated only by using indirect immunofluorescence technique on monkey esophageal epithelium. The determination of human antibody reactive against TGt-2 has the same diagnostic value as the EMA, but has a greater clinical utility, because it can be achieved with simpler techniques (commercial ELISA). Such diagnostic methods were found to allow for high sensitivity and specificity (close to 90\%) in the various studies carried out in the early years after their commercialization [25].

As the determination of anti-TGt-2 antibodies became used more routinely, a marked decrease of sensitivity was found to exist when studying patients without villous atrophy (or with a mild degree, as normally happens in adults). Several published studies among large groups of celiac patients, have clearly demonstrated that a positive anti-TGt-2 antibody test, correlates linearly with the degree of severity of the histological duodenal lesions found. In patients presenting only lymphocytic enteritis (Marsh I), its sensitivity is very low, between $15-30 \%$ at best [26-28]. These findings have led to the application of new diagnostic strategies, such as the concurrent use of genetic testing, or the determination of anti-TGt-2 antibodies in duodenal aspirate in cases of negative serology. An alternative approach to increasing sensitivity relies on the use of a corrected threshold for positivity; its value has been recommended as $2 \mathrm{U} / \mathrm{ml}$ for adults [15,29]. This is the threshold that we have applied in the present study. We did not employ EMA antibodies, because they measure the same substract than the anti-TGt-2 and the anti-gliadin, because they have a lower sensitivity and specificity than the anti-TGt-2 used.

The findings from duodenal biopsies are still considered by most experts as the "cornerstone" or the "gold standard" for diagnosis of CD. However, this statement emphatic as it may sound, is currently being challenged, especially if we take into account the adult forms of $\mathrm{CD}$. In cases with very high levels of anti-TGt-2 antibodies $(>100 \mathrm{U} / \mathrm{ml})$, the duodenal biopsy may be avoided, since in most cases (>90\%) such high levels are associated with the presence of villous atrophy [30]. When the histological results are normal, but the serology is positive and the clinical picture is suggestive of $\mathrm{CD}$, the diagnostic uncertainty remains, and in such cases a gluten-free diet (GFD) could be recommended for at least 6 months, before definitely ruling out a $\mathrm{CD}$.

When this diagnosis is dubious, it could be useful to perform a genetic and familial study such as those we carried out in the present study. While the prevalence of DQ2 in the general population is $20-40 \%$, it reaches $90 \%$ among celiac patients, as confirmed in a study by FernándezBañares et al., in which a series of patients with presumed irritable bowel syndrome and predominant diarrhea were screened for possible CD [31]. The determination of the known genetic markers for celiac disease (DQ2 and DQ8) therefore, has a high negative predictive value.

In the present study we have found a high prevalence of celiac disease among MS patients (11.1\%) based mainly on the presence of villous atrophy in the duodenal biopsy. This is between 5-10 times higher that the frequency found in the general population. All the 8 celiac patients were female. Six had gastrointestinal symptoms, with predominant constipation in 5 and chronic gastro-esophageal reflux disease (GERD), in 3. Surprisingly only one patient had chronic diarrhoea and weight loss. This form is called "atypical presentation" and is common in adults, in contrast to the "classical form" that predominates in children. The response of the gluten-free diet was excellent in all of them, both from the digestive and the neurologic point of view in the average follow-up period of three years long.

The predominant neurologic clinical presentation on these celiac patients was myelitis in 4 , and mixed in the rest. No ataxic forms were found. No specific findings were seen on their MRI's compared with non-celiac patients.

Only 3 celiac patients were treated with IFN beta 1-b, and we didn't find any more autoimmune disturbances in them. The remaining $5 \mathrm{CD}$ patients, were only on a GFD.

We performed a family screening study in all the $72 \mathrm{MS}$ patients, not only in the 8 celiacs, and we found a very high prevalence of $C D$ among them, with a total of 23 first degree relatives positive among 126 screened (32\%)

$\mathrm{CD}$ is also associated with several neurological conditions at a significantly higher frequency than in the general population, as can be observed in patients with migraine [32,33]. Cerebellar ataxia associated with gluten is the syndrome most commonly associated with $\mathrm{CD}$, especially in adult patients. Many immunopathogenic mechanisms and different antibodies associated with gluten have been described which are capable of crossing the blood-brain barrier and deposit at the level of the Purkinje cells, where they produce a marked inflammatory response followed by neuronal degeneration and cerebellar atrophy $[34,35]$. An isoenzyme of TGt has been recently described, specifically the subtype 6, which is present in the cerebellum of patients with $\mathrm{CD}$-associated ataxia, and its positivity would be useful in explaining the pathogenesis of this process [36].

\section{Conclusions}

In this prospective series of 72 RRMS patients, we have found at least $8(11.1 \%)$ with a related $C D$, confirmed by the presence of serological antibodies (TGt-2) in 7 and villous atrophy in all of them. This represents a 
prevalence of gluten intolerance 5.5 to 11 times higher in this group of patients than that found of the general population.

A gluten-free diet should be considered for any RRMS patient, who exhibits these serological and histological findings, characteristic of the presence of an associated celiac disease.

\section{Acknowledgements}

The authors would like to thank Marino Santirso for the linguistic review of the final manuscript. We would like also to express our gratitude to Mr. John Hardwick for checking the English text.

This paper has been sponsored for his publication fees by the FICYT (OIB) from the Regional Research Agency of Asturias (Spain).

\section{Author details}

${ }^{1}$ Gastroenterology, Hospital Universitario Central de Asturias (HUCA), C/ Celestino Villamil, s. $n^{\circ}$., 33006. Oviedo, Spain. ${ }^{2}$ Neurology, Hospital Universitario Central de Asturias (HUCA), c/Celestino Villamil, s. $n^{\circ}$., 33006. Oviedo, Spain. ${ }^{3}$ mmmunology Services Hospital Universitario Central de Asturias (HUCA), c/Celestino Villamil, s. nº., 33006. Oviedo, Spain.

\section{Authors' contributions}

Conception and design: $\mathrm{LR}$ and $\mathrm{CHL}$. The analysis and interpretation of the data: LR and SG. Drafting of the article: DF, NA, ALV, SG. Critical revision of the paper, for important intellectual content: LR, and CHL. All authors read and approved the final manuscript

\section{Competing interests}

The authors declare there are no competing interests.

Received: 31 October 2010 Accepted: 7 March 2011

Published: 7 March 2011

\section{References}

1. Compston A, Coles A: Multiple sclerosis. Lancet 2008, 372:1.502-17.

2. Chandran $S$, Hunt $D$, Joannides $A$, et al: Myelin repair: the role of stem and precursor cells in multiple sclerosis. Philos Trans $R$ Soc London Biol Sci 2008, 363:171-83.

3. Sawcer S, Compston A: Multiple sclerosis: Light at the end of the tunnel. Eur J Hum Genet 2006, 14:257-8.

4. Tsutsui S, Stys PK: Degeneration versus autoimmunity in Multiple Sclerosis. Ann Neurol 2009, 66:711-3.

5. Lincoln JA, Cook SD: An overview of gene-epigenetic-environmental contributions to MS causation. J Neurol Sci 2009, 286:54-7.

6. Tintore M, Rovira A, Río J, et al: New diagnostic criteria for multiple sclerosis: application in first demyelinating episode. Neurology 2003, 60:27-30.

7. Carrol WM: Oral Therapy for Multiple Sclerosis. N Engl J Med 2010, 362:456-8.

8. James MB, Scott BB: Celiac disease: the cause of the various associated disorders? Eur J Gastroenterol Hepatol 2001, 13:1119-21.

9. Rodrigo L: Celiac disease. World J Gastroenterol 2006, 12:6585-93.

10. Green PHR, Cellier C: Celiac disease. N Engl J Med 2007, 357:1731-43.

11. Kakar S, Nehra V, Murray JA, et al: Significance of intraepithelial lymphocytosis in small biopsy samples with normal mucosal architecture. Am J Gastroenterol 2003, 98:2027-33.

12. Shor $\mathrm{DB}$, Barzilai $\mathrm{O}$, Ram M, et al: Gluten sensitivity in multiple sclerosis: experimental myth or clinical truth? Ann N Y Acad Sci 2009, 1173:343-9.

13. Polman $\mathrm{CH}$, Reingold SC, Edan G, et al: Diagnostic criteria for multiple sclerosis: 2005 revisions to the "Mac Donald Criteria". Ann Neurol 2005, 58:840-6.

14. Marine $M$, Fernández-Bañares $F$, Alsina $M$, et al: Impact of mass screening for gluten-sensitive enteropathy in working population. World $\rfloor$ Gastroenterol 2009, 15:1331-8.
15. Fernández $E$, Riestra $S$, Rodrigo $L$, et al: Comparison of six human antitransglutaminase ELISA-tests in the diagnosis of celiac disease in the Saharawi population. World J Gastroenterol 2005, 11:3762-6.

16. Marsh MN: Gluten, major histocompatibility complex and the small intestine. A molecular and immunobiologic approach to the spectrum of gluten sensitivity ('celiac sprue'). Gastroenterology 1992, 102:330-54.

17. Oberhuber G, Granditsch G, Vogelsang H: The histopathology of coeliac disease. Time for a standardized report scheme for pathologists. Eur J Gastroenterol Hepatol 1999, 11:1185-94.

18. Hadjivassiliou M, Sanders DS, Grünewald RA, et al: Gluten sensitivity: from gut to brain. Lancet Neurol 2010, 9:318-30.

19. Kom T: Pathophysiology of multiple sclerosis. J Neurol 2008, 255(Suppl 6):2-6.

20. López-Vázquez A, Rodrigo L, Fuentes $D$, et al: MHC class I chain related gene A (MICA) modulates the development of coeliac disease in patients with the high risk heterodimer DQA1*0501/DQB1*0201. Gut 2002, 50:336-40.

21. Fasano A, Berti I, Gerarduzzi T, et al: Prevalence of celiac disease in at-risk and not-at-risk groups in the United States: a large multicenter study. Arch Intern Med 2003, 163:286-92.

22. Shan L, Molberg $\varnothing$, Parrot I, et al: Structural basis for gluten intolerance in celiac sprue. Science 2002, 297:2275-9.

23. Molberg O, McAdam SN, Sollid LM: Role of tissue transglutaminase in celiac disease. J Pediatr Gastroenterol Nutr 2000, 30:232-40.

24. Maiuri $L$, Ciacci $C$, Ricciardelli I, et al: Association between innate response to gliadin and activation of pathogenic T cells in celiac disease. Lancet 2003, 362:30-7.

25. Dieterich W, Ehnis T, Bauer M, et al: Identification of tissue transglutaminase as the auto-antigen of celiac disease. Nat Med 1997, 3:797-801

26. Tursi A, Brandimarte G, Giorgetti GM: Prevalence of anti-tissue transglutaminase antibodies in different degrees of intestinal damage in celiac disease. J Clin Gastroenterol 2003, 36:219-21.

27. Esteve $M$, Rosinach $M$, Fernández-Bañares $F$, et al: Spectrum of gluten sensitive enteropathy in first-degree relatives of coeliac patients:clinical relevance of lymphocytic enteritis. Gut 2006, 55:1739-45.

28. Dickey W, Hughes DF, McMillan SA: Disappearance of endomysial antibodies in treated celiac disease does not indicate histological recovery. Am J Gastroenterol 2000, 95:712-4.

29. Santaolalla R, Fernández-Bañares $F$, Rodríguez $R$, et al: Diagnostic value of duodenal anti-tissue transglutaminase antibodies in gluten-sensitive enteropathy. Aliment Pharmacol Ther 2008, 27:820-9.

30. Vivas S, Ruiz de Morales JG, Riestra S, et al: Duodenal biopsy may be avoided when high transglutaminase antibody titers are present. World J Gastroenterol 2009, 15:4775-80.

31. Fernández-Bañares F, Esteve M, Salas A, et al: Systematic evaluation of causes of chronic watery diarrea with functional characteristics. Am J Gastroenterol 2007, 102(11):2520-2528.

32. Roche Herrero MC, Arcas Martínez J, Martínez-Bermejo A, et al: The prevalence of headache in a population of patients with coeliac disease. Rev Neurol 2001, 32:301-9.

33. Zelnik N, Pacht T, Obeid R, et al: Range of neurologic disorders in patients wih celiac disease. Pediatrics 2004, 113:1672-6.

34. Bushara KO: Neurologic presentation of celiac disease. Gastroenterology 2005, 128:S92-7.

35. Wong $D$, Dwinnel $M$, Schulzer $M$, et al: Ataxia and the role of antigliadin antibodies. Can J Neurol Sci 2007, 34:193-6.

36. Hadjivassiliou M, Aeschlimann P, Strigun A, et al: Autoantibodies in gluten ataxia recognize a novel neuronal transglutaminase. Ann Neurol 2008, 64:332-43.

Pre-publication history

The pre-publication history for this paper can be accessed here: http://www.biomedcentral.com/1471-2377/11/31/prepub

doi:10.1186/1471-2377-11-31

Cite this article as: Rodrigo et al:: Prevalence of celiac disease in multiple sclerosis. BMC Neurology 2011 11:31. 